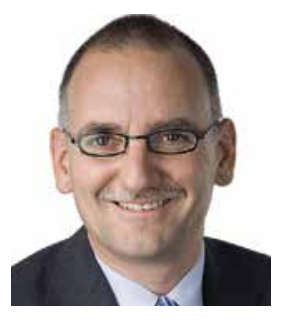

Prof. Dr. Felix J.F. Herth

felix.herth@thoraxklinik-heidelberg.de

\title{
Information kompakt - im Trend der Zeit
}

Die Pneumologie befindet sich im Aufwind: Lungenerkrankungen nehmen im Verhältnis zu anderen Erkrankungen der Inneren Medizin überproportional zu. Erkrankungen der Lunge und/oder der Atemwege sind bezüglich Morbidität, Mortalität und Kosten von großer Bedeutung. So weisen Prognosen für die weltweite Mortalitätsentwicklung aus, dass 4 der 10 häufigsten zum Tode führenden Erkrankungen unser Fachgebiet betreffen. Die Bedeutung einzelner pneumologischer Krankheiten für die Gesundheitsversorgung zeigt sich besonders deutlich anhand der prognostizierten prozentualen Zunahme von Lungenkrankheiten bis 2050. Diese Zahlen geben einen wichtigen Hinweis auf den zu erwartenden organisatorischen, personellen und finanziellen Bedarf im Bereich der qualifizierten pneumologischen Versorgung.

Aus diesen Gründen nehmen die Forschungsaktivitäten in diesem Gebiet in den letzten Jahren zu. Gibt man in gängige Suchmaschinen wie z.B. PubMed Stichworte wie «pulmonary medicine» oder «lung diseases» ein, zeigt die Zahl der angegebenen Literaturstellen wöchentlich eine Steigerung im knapp dreistelligen Bereich. So werden derzeit schon mehr als 750000 Artikel aufgelistet.

Nicht nur gegenüber unseren Patienten, sondern auch qua Gesetz sind wir zur Fort- und Weiterbildung verpflichtet. Wie neues Wissen erworben wird, ist letztlich jedem Einzelnen überlassen.

Wie soll man die Flut der Informationen überblicken? Karger Kompass schlägt hier einen Weg ein, der das Wissen aus ver- schiedenen Karger-Publikationen eines Fachgebiets bündelt und kompakt verfügbar macht. Ausgewählte internationale Publikationen werden durch Experten aufbereitet, d.h., die wichtigsten Aspekte eines Artikels werden dargestellt und anschließend kommentiert. Ein besonderes Augenmerk wird dabei auf die Vermittlung von praxisrelevantem Wissen gelegt. Die Kommentatoren nutzen jedoch auch die Gelegenheit, die Publikationen kritisch zu hinterfragen und auf deutsche Verhältnisse zu übertragen: Ist eine Kernaussage so wirklich zu bestätigen? Wie muss sie im Verhältnis zu bisherigen Erkenntnissen gewertet werden? Was bedeutet dies für die Patienten? Die Kommentare erfolgen sachlich korrekt, produktneutral und ohne jegliche Beeinflussung, damit Karger Kompass dem hohen Anspruch eines Wissenstransfers aus internationalen Publikationen in den medizinischen Praxisalltag gerecht wird. Zweimal im Jahr wird im Rahmen von Karger Kompass eine neue Publikation zum Fachgebiet Pneumologie erscheinen. Wir sind überzeugt, dass diese Art des Wissenstransfers Erfolg hat, weil sie Kliniker und niedergelassene Fachärzte in die Lage versetzt, sich schnell, aber dennoch umfassend über wichtige Erkenntnisse und Entwicklungen in ihrem Fachgebiet zu informieren.

Ihnen, liebe Leser, viel Spaß und reichlich Informationsgewinn bei der Lektüre der hier vorliegenden ersten Ausgabe.

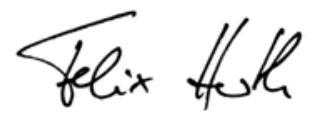

\section{KARGER \\ Fax +497614520714 \\ information@karger.com} www.karger.com
(C) 2013 S. Karger AG, Basel

2296-0368/13/0011-0007\$38.00/0

Accessible online at: www.karger.com/kom
Prof. Dr. med. Felix Herth

Pneumologie und Beatmungsmedizin

Thoraxklinik, Universitätsklinik Heidelberg

Amalienstraße 5, 69126 Heidelberg, Deutschland 